\title{
Abuso Sexual Infantil, Câncer e Outras Doenças Avaliados pelo Rorschach: Revisão Sistemática
}

\author{
Eduardo dos Santos de Lima ${ }^{1}$ \\ ${ }^{1}$ Universidade de Passo Fundo, Passo Fundo, RS, Brasil.
}

\author{
Silvana Alba Scortegagna ${ }^{1}$ \\ ${ }^{1}$ Universidade de Passo Fundo, Passo Fundo, RS, Brasil.
}

Resumo: Algumas vivências traumáticas podem suscitar alterações autoperceptivas, afetivas e cognitivas, comuns em casos procedentes dos contextos forense e clínico, o que denota a importância de verificar a origem motivadora dessas alterações. Esta revisão de literatura buscou identificar as variáveis compartilhadas e discrepantes do método de Rorschach nas avaliações de vítimas de abuso sexual e de pacientes com câncer e enfermidades do corpo. Com base no método Preferred Reporting Items for Systematic Reviews and Meta-Analyses (PRISMA), foram encontrados 11 artigos publicados entre 2008 e 2018, dos quais quatro concernem avaliações de vítimas de abuso sexual; seis, de pacientes com doenças do corpo; e um, de paciente com câncer. As variáveis compartilhadas foram: autopercepção (MOR), relacionamento interpessoal (PER, PHR, T, AG), afeto (SumC', SumY) e ajuste perceptivo (FQ-). Vítimas de abuso sexual diferiram em: autopercepção (An), relacionamento interpessoal (GHR), ideação (m, PTI) e alguns conteúdos traumáticos (Bl e Sx). Pacientes com câncer e doenças do corpo diferenciaram-se em: relacionamento interpessoal (CDI, FD), afeto (CF+C > FC, SumV), ideação (Mp, WSum6, ALOG, DV, DR), processamento (Zd, DQv, PSV, Dd), mediação (P, S-, X-\%, Xu\%) e controle (D, AdjD, es). Os resultados indicam o protagonismo do corpo, da autopercepção prejudicada e de sentimentos depressivos e de ansiedade. Em vítimas de abuso sexual, o corpo sinaliza marcas de vivências traumáticas, enquanto em pacientes com câncer e outras doenças, o corpo denuncia sentimentos de angústia e de morte. Os resultados contribuem para auxiliar nas avaliações e intervenções psicológicas dirigidas a essa população.

Palavras-chave: Avaliação Psicológica, Método Projetivo, Trauma Psíquico.

\section{Child Sexual Abuse, Cancer, and Other Diseases Assessed by the Rorschach: Systematic Review}

\begin{abstract}
Some traumatic experiences arising from the forensic and clinical context may lead to self-perceptive, affective, and cognitive alterations, indicating the importance of verifying the origin that motivated these changes. This literature review aimed to identify the shared and discrepant variables of the Rorschach method in assessing victims of sexual abuse and patients with cancer and body diseases. Based on the Preferred Reporting Items for Systematic Reviews and Meta-Analyzes (PRISMA), the review found 11 articles published between 2008 and 2018: four about victims of sexual abuse, six about patients with body diseases, and one about cancer patient. The shared variables were self-perception (MOR), interpersonal relationship (PER, PHR, T, AG), affection (SumC', SumY), and perceptual adjustment (FQ-). Victims of sexual abuse differed in regard to self-perception (An), interpersonal relationship (GHR), ideation (M, PTI), and some traumatic content ( $\mathrm{Bl}$ and $\mathrm{Sx}$ ). In turn, patients with cancer and body diseases differed regarding interpersonal relationship (CDI, FD), affection (CF + C > FC, SumV), ideation (Mp, WSum6, ALOG, DV, DR), processing (Zd, DQv, PSV and Dd), mediation (P, S-, X-\% and Xu\%), and control (D, AdjD, es). The shared results indicate the prominence of the body, the impaired
\end{abstract}


self-perception, and feelings of depression and anxiety. In victims of sexual abuse, the body signaled marks of traumatic experiences, whereas in patients with cancer and other diseases it indicates feelings of anguish and death. This study contributes to assisting in evaluations and psychological interventions aimed at this population.

Keywords: Psychological Assessment, Projective Method, Psychic Trauma.

\title{
Abuso Sexual Infantil, Cáncer y Otras Enfermedades Evaluadas por el Rorschach: Revisión Sistemática
}

\begin{abstract}
Resumen: Algunas experiencias traumáticas pueden provocar alteraciones autoperceptivas, afectivas y cognitivas, comunes en casos originados por el contexto forense y clínico; por eso, la importancia de verificar el origen que motivó estos cambios. Esta revisión de la literatura trató de identificar las variables compartidas y discretantes del método Rorschach en las evaluaciones de las víctimas de abuso sexual y de pacientes con cáncer y enfermedades corporales. Sobre la base de Preferred Reporting Items for Systematic Reviews and Meta-Analyses (PRISMA), se encontraron 11 artículos publicados entre 2008 y 2018 ; de los cuales cuatro son sobre evaluaciones de víctimas de abuso sexual, seis sobre pacientes con enfermedades corporales y uno sobre paciente con cáncer. Las variables compartidas fueron: autopercepción (MOR), relación interpersonal (PER, PHR, T, AG), afecto (SumC, SumY) y ajuste perceptual (FQ-). Las víctimas de abuso sexual diferían en: autopercepción (An), relación interpersonal (GHR), ideación (m, PTI), y algo de contenido traumático ( $\mathrm{Sx}$ y Bl). Los pacientes con cáncer y enfermedades corporales se diferenciaron en: relación interpersonal (CDI, FD), afecto (CF+C> FC, SumV), ideación (Mp, WSum6, ALOG, DV, DR), procesamiento (Zd, DQv, PSV y Dd), mediación (P, S-, X-\% y Xu\%) y control (D, AdjD, es). Los resultados indican el protagonismo del cuerpo, la autopercepción dañada y los sentimientos depresivos y de ansiedad. En las víctimas de abuso sexual, el cuerpo señala marcas de experiencias traumáticas, mientras que en los pacientes con cáncer y otras enfermedades el cuerpo denuncia sentimientos de angustia y muerte. En conclusión, los resultados contribuyen a ayudar en las evaluaciones e intervenciones psicológicas dirigidas a esta población.
\end{abstract}

Palabras clave: Evaluación Psicológica, Método Proyectivo, Trauma Psíquico.

\section{Introdução}

O abuso sexual contra crianças e adolescentes é um problema de saúde pública. De 2011 a 2017 foram notificados 184.524 casos de abuso sexual, dos quais $45 \%(\mathrm{n}=83.068)$ foram praticados contra adolescentes de 10 a 19 anos e $31,5 \%(n=58.037)$, contra crianças de até 9 anos de idade. Dessas crianças, $74,2 \%(n=43.034)$ eram do sexo feminino e $25,8 \%(n=14.996)$ eram do sexo masculino. Das notificações de abuso sexual contra crianças, $69,2 \%(\mathrm{n}=40.154)$ ocorreram nas residências e em $37 \%(n=19.542)$ os abusos foram recorrentes (Ministério da Saúde [MS], 2018).

São consideradas abuso sexual infantil as interações na qual a criança ou o adolescente é usado para satisfação sexual de um adulto ou de outro adolescente em fase psicossexual mais avançada. As interações abusivas abrangem casos de assédio, estupro, pornografia infantil, exploração sexual, abuso incestuoso, práticas eróticas, pedofilia, voyeurismo, manuseio, penetração com pênis ou objetos, exibicionismo e interação forçada com materiais pornográficos (Krindges, Macedo, \& Habigzang, 2016; MS, 2018).

O abuso sexual infantil apresenta características próprias que o diferem de outras formas de abuso. O uso de ameaças, a imposição de segredos e a dificuldade de encontrar testemunhas (Krindges et al., 2016; Schaefer, Brunnet, Lobo, Carvalho, \& Kristensen, 2018) facilitam o abuso continuado da vítima. Somadas a isso, as características cognitivas e afetivas próprias da infância, em estágios de desenvolvimento 
anteriores às do adulto, e a vulnerabilidade dessas vítimas podem fazer com que esse evento traumático gere prejuízos ao desenvolvimento biopsicossocial das vítimas, em todo o processo de envelhecimento.

A emergência de sintomas depressivos é comumente acompanhada de ansiedade, estresse pós-traumático, comportamentos disfuncionais e autodestrutivos, perturbações sexuais, transtornos somáticos e dificuldade de relacionamento (Schaefer et al., 2018). Em relação à severidade dos efeitos do abuso nas vítimas, sabe-se que eles serão influenciados por fatores como a idade da criança, os tempos de início e duração do abuso, os diferentes tipos de violência aplicadas e o tipo de vínculo com o abusador (Florentino, 2015).

A psicologia, nesse contexto, pode assumir um papel de especial relevância tanto no âmbito forense, ao responder às demandas de avaliação de crianças com suspeita de abuso ou com abuso comprovado (Cadan \& Albanese, 2018), quanto no clínico, ao prover avaliação e atendimento psicológico às vítimas (Rossato, Santeiro, Barroso, \& Scorsolini-Comin, 2018). A intervenção profissional tende a reduzir os efeitos danosos físicos e psicossociais, mesmo quando tardia (Hohendorff, Habigzang, \& Koller, 2015; Lahav \& Elklit, 2016), mas quanto mais precocemente for comprovada a ocorrência da vitimização sexual e iniciado tratamento apropriado, menores serão os efeitos desse infortúnio. Surge, então, a necessidade de instrumentos que apresentem propriedades psicométricas e evidências de validade para avaliar o funcionamento mental de crianças e adolescentes vítimas de abuso sexual, por meio dos quais os profissionais da psicologia possam aferir interpretações e proceder encaminhamentos com segurança.

O método de Rorschach, uma técnica mundialmente conhecida, pode ser útil nesse contexto. Entre as principais razões do uso dessa ferramenta avaliativa da personalidade, destaca-se que ela oferece aspectos relacionados à percepção, isto é, a como as pessoas compreendem e interpretam os fatos (Meyer, 2017; Meyer \& Eblin 2012; Mihura, Meyer, Dumitrascu, \& Bombel, 2013); proporciona dados sobre a organização e a sofisticação do processamento das informações; e reúne sensibilidade para avaliar representações pessoais de considerável conteúdo idiossincrático (Meyer \& Eblin, 2012).

Alguns estudos mostraram que o método de Rorschach no Sistema Compreensivo (RSC) foi útil para diferenciar vítimas de abuso sexual quando comparadas com grupos de não-vítimas (Scortegagna \& Villemor-Amaral, 2009, 2013; Scortegagna, Ribeiro, \& Villemor-Amaral, 2016). Entre os achados, o estudo de Scortegagna e Villemor-Amaral (2009) indicou que vítimas de abuso sexual apresentaram aumento na média das variáveis que sinalizam pensamentos intrusivos referentes às experiências traumáticas vivenciadas e preocupação com o próprio corpo $(\mathrm{m}=2$, $\mathrm{Bl}=0,8, \mathrm{An}=1,58, \mathrm{MOR}=1,66$ ), além de predisposição para percepções mais equivocadas (FQ- $=7,6)$ quando comparadas às não-vítimas $(\mathrm{m}=1,6, \mathrm{Bl}=0,2$, An $=0,9, \mathrm{MOR}=0,85, \mathrm{FQ}-=5,6)$. O estudo de Perfect, Tharinger, Keith e Lyle-Lahroud (2011) revelou que vítimas de violência sexual apresentavam comportamentos mais patológicos quanto ao estresse emocional (SumY = 0,96, SumC' $=1,01, T=0,36$ ), perturbações do pensamento e da percepção (PTI $=0,45$ ) e mais tendência à compreensão problemática ou menos adaptativa das interações interpessoais (PHR > GHR).

Similarmente, pessoas que enfrentam o câncer podem ter alterações de pensamento e percepção, principalmente em relação a sua autoimagem. O câncer, como invasor traumático, pode causar danos e suscitar sentimentos de aniquilamento do corpo e de incongruência corporal. Signorelli (2015) analisou protocolos do Rorschach pela Escola Francesa, de 15 mulheres, entre 36 e 66 anos de idade, com diferentes tipos de câncer. As respostas registradas faziam referência a aspectos físicos em corpos que assumiam limites inadequados, formas imprecisas ou expressões verbais do próprio câncer, o que, segundo a autora, podem ser entendidos como expressão da aflição sentida pelos pacientes. Essa invasão traumática tem semelhanças com o abuso sexual infantil, embora de ordem distinta.

Contudo, estudos que utilizam o RSC em crianças com câncer são inexistentes. Recentemente, Lima (2020) buscou evidências de validade para o método de Zulliger no Sistema Compreensivo (ZSC), utilizando a otimização de respostas na avaliação de crianças vítimas de violência sexual, que foram comparadas a crianças com câncer e crianças de grupo normativo. As hipóteses testadas relacionavam-se ao aumento de indicadores do ZSC que apontavam problemas de relacionamento interpessoal, de autoimagem e de conteúdo traumático, em crianças vítimas de violência sexual. Anterior a esse trabalho, até o presente momento e que se tenha conhecimento, apenas o estudo de Goggin, Lansky e Hassanein (1976) avaliou crianças com câncer utilizando o método de Zulliger. 
A partir da hipótese de que crianças vítimas de violência sexual e pacientes com câncer podem apresentar semelhanças nas respostas do RSC, ampliar essa questão para outras doenças que também possam causar traumatismos psíquicos se faz necessário. Por exemplo, o estudo de Balottin et al., (2018) utilizando o RSC, indicou que pacientes com enxaqueca apresentavam visão negativa de si e problemas de relacionamentos interpessoais (PER, MOR e FD). $O$ estudo ainda sugere que $\mathrm{o}$ isolamento social pode favorecer a percepção da dor e o aumento da preocupação com seus próprios corpos.

Indicadores discriminantes de vítimas de abuso sexual infantil e de não-vítimas, observados no método de Rorschach, podem também ser representativos do sofrimento de pessoas que não tenham sofrido violência sexual, mas que tenham sido vitimizadas por traumas físicos como o câncer ou outras doenças. Nesse contexto, é importante conhecer as variáveis representativas de cada uma das situações vivenciadas para que se possa realizar avaliações respaldadas em bases empíricas. Com esse propósito, este estudo objetivou identificar as variáveis compartilhadas e discrepantes do método de Rorschach nas avaliações de vítimas de abuso sexual e de pacientes com câncer e/ou doenças do corpo.

\section{Método}

Com base nas orientações do Preferred Reporting Items for Systematic Reviews and Meta-Analyses PRISMA (Moher, Liberati, Tetzlaff, Altman, \& The PRISMA Group, 2009), realizou-se revisão dos artigos publicados que utilizaram o método de Rorschach com vítimas de abuso sexual infantil e com pacientes com câncer e/ou doenças físicas. Selecionou-se artigos nas bases de dados da CAPES - Scielo, PubMed, Pepsico e, Science Direct, APA - publicados nos anos de 2008 a 2018. Utilizou-se como descritores "Rorschach and sexual abuse", "Rorschach and cancer" e "Rorschach and physical illness". Como critérios de elegibilidade contemplou-se estudos de crianças que sofreram violência sexual e pacientes com doenças do corpo, como o câncer. Excluiu-se estudos com adultos vítimas de abuso sexual, estudos que não utilizaram o método de Rorschach nas avaliações, artigos duplicados, dissertações e teses. Os títulos e resumos de todos os artigos resultantes da busca foram avaliados. Leu-se na íntegra os artigos cujos resumos não ofereceram todas as informações necessárias para sua inclusão ou exclusão.

\section{Resultados}

As buscas nas bases de dados totalizaram 760 artigos. Aplicando os critérios de elegibilidade e de exclusão, selecionou-se 11 artigos. A Figura 1 mostra o processo de seleção e exclusão dos textos, a partir dos termos utilizados.

A seguir, dividiu-se os artigos em dois eixos temáticos. O primeiro eixo temático abordou os artigos que utilizaram o método de Rorschach na avaliação de vítimas de abuso sexual infantil; o segundo eixo temático reuniu os artigos que abordaram o método de Rorschach na avaliação de pacientes com câncer e outras doenças físicas. A Tabela 1 apresenta os artigos que utilizaram o método de Rorschach na avaliação de vítimas de abuso sexual infantil e doenças do corpo, contemplando autores, ano, objetivo do estudo, população investigada, sistema utilizado/ variáveis selecionadas e resultados.
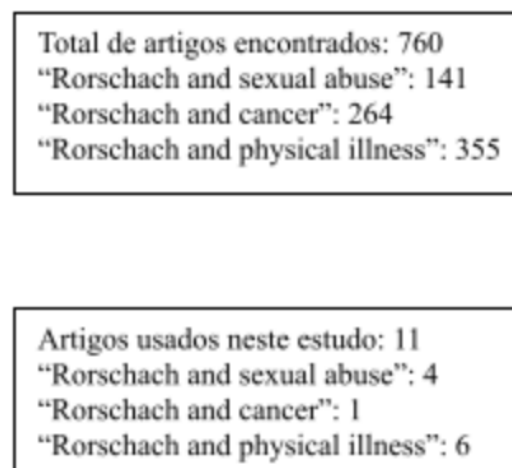

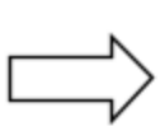

Artigos duplicados: 8

"Rorschach and sexual abuse": 1

"Rorschach and cancer": 2

"Rorschach and physical illness": 5

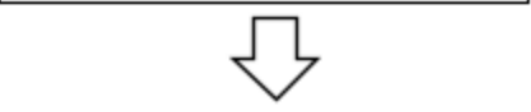

Artigos removidos por incoerência

com o tema: 741

"Rorschach and sexual abuse": 136

"Rorschach and cancer": 261

"Rorschach and physical illness": 344

Figura 1

Seleção dos artigos para análise. 
Tabela 1

Descrição de estudos com o Método de Rorschach sobre abuso sexual infantil e doenças do corpo (2008-2018).

\begin{tabular}{|c|c|c|c|c|}
\hline $\begin{array}{c}\text { Autor/ano/ } \\
\text { local }\end{array}$ & Objetivo & População & Variáveis & Resultados \\
\hline \multicolumn{5}{|c|}{ Abuso Sexual Infantil } \\
\hline $\begin{array}{l}\text { Scortegagna \& } \\
\text { Villemor- } \\
\text { Amaral, 2013/ } \\
\text { Brasil }\end{array}$ & $\begin{array}{l}\text { Comparar as respostas } \\
\text { de movimento } \\
\text { inanimado (m) e } \\
\text { conteúdo de sangue }(\mathrm{Bl}) \\
\text { emitidas nos protocolos } \\
\text { do RSC, entre vítimas } \\
\text { de abuso sexual e não } \\
\text { vítimas. }\end{array}$ & $\begin{array}{l}29 \text { crianças entre } \\
10 \text { e } 14 \text { anos } \\
\text { cursando ensino } \\
\text { fundamental e } \\
\text { médio, divididas } \\
\text { em } 2 \text { grupos, um } \\
\text { de vítimas e outro } \\
\text { de não-vítimas. }\end{array}$ & $\begin{array}{c}\text { Bl, An, Sx, m, } \\
\text { SumC', SumY, AG, } \\
\text { MOR. }\end{array}$ & $\begin{array}{c}\text { Vítimas de abuso } \\
\text { sexual apresentaram } \\
\text { sentimentos de } \\
\text { desamparo e } \\
\text { impotência(m); } \\
\text { associadas a conteúdos } \\
\text { mais violentos e } \\
\text { destrutivos (Bl), com } \\
\text { características } \\
\text { mórbidas (MOR). }\end{array}$ \\
\hline $\begin{array}{l}\text { Barnett, } \\
\text { Heinze, \& } \\
\text { Arble, 2013/ } \\
\text { EUA }\end{array}$ & $\begin{array}{l}\text { Estudo longitudinal } \\
\text { buscou avaliar se o RSC } \\
\text { era capaz de avaliar } \\
\text { processamento de } \\
\text { informações e sintomas } \\
\text { de depressão infantil } \\
\text { após } 15 \text { meses. }\end{array}$ & $\begin{array}{c}44 \text { crianças vítimas } \\
\text { de abuso sexual, } \\
\text { entre } 6 \text { e } 15 \text { anos, } \\
\text { na primeira } \\
\text { avaliação. }\end{array}$ & $\begin{array}{l}\text { Sum de FM, m, } \\
\text { SumC', SumT, } \\
\text { SumY, SumV, S, } \\
\text { MOR, Score Z, Bl, } \\
\text { Sx, An, DEPI, AG. }\end{array}$ & $\begin{array}{l}\text { O índice de conteúdo } \\
\text { de trauma foi } \\
\text { significativamente } \\
\text { correlacionado a } \\
\text { depressão (Bl, Sx, An, } \\
\text { MOR, AG). }\end{array}$ \\
\hline $\begin{array}{c}\text { Perfect, } \\
\text { Tharinger, } \\
\text { Keith, \& } \\
\text { Lyle-Lahroud, } \\
\text { 2011/ } \\
\text { EUA }\end{array}$ & $\begin{array}{l}\text { Avaliar se as variáveis } \\
\text { do RSC e a escala do } \\
\text { Minnesota Multiphasic } \\
\text { Personality Inventory } \\
\text { (MMPI)-A que poderiam } \\
\text { indicar severidade dos } \\
\text { subtipos de maus-tratos. }\end{array}$ & $\begin{array}{l}157 \text { adolescentes, } \\
\text { de ambos os sexos } \\
\text { e idades entre } 14 \\
\text { e } 17 \text { anos, com } \\
\text { histórico de maus- } \\
\text { tratos. }\end{array}$ & $\begin{array}{c}\text { PER, MOR, Ego, } \\
\text { SumY, SumC', } \\
\text { DEPI, Afr, PTI, } \\
\text { WSum6, X-\%, } \\
\text { Isol, H, e Textura. }\end{array}$ & $\begin{array}{l}\text { As variáveis MOR, PER, } \\
\text { SumY, SumC', PTI, GHR, } \\
\text { PHR e SumT foram } \\
\text { significativamente } \\
\text { associadlas a severidade } \\
\text { do abuso sexual. }\end{array}$ \\
\hline $\begin{array}{l}\text { Scortegagna \& } \\
\text { Villemor- } \\
\text { Amaral, 2009/ } \\
\text { Brasil }\end{array}$ & $\begin{array}{c}\text { Buscar evidências de } \\
\text { validade do RSC em } \\
\text { vítimas de abuso sexual. }\end{array}$ & $\begin{array}{l}76 \text { indivíduos, de } \\
\text { ambos os sexos, } \\
\text { de } 10 \text { a } 14 \text { anos, } \\
\text { cursando ensino } \\
\text { fundamental e } \\
\text { médio, divididos } \\
\text { em dois grupos, } \\
\text { um de vítimas } \\
\text { e outro de não- } \\
\text { vítimas }\end{array}$ & $\begin{array}{l}\text { EgoIndex, [H: } \\
(\mathrm{H})+\mathrm{Hd}+(\mathrm{Hd})] \\
\text { CO e AG e os } \\
\text { índices GHR e } \\
\text { PHR, Xo\%, Xu\% } \\
\text { e X-\%, m, SumY, } \\
\text { SumC'. }\end{array}$ & $\begin{array}{c}\text { Houve diferenças } \\
\text { significativas nas } \\
\text { respostas m e de conteúdo } \\
\text { Bl e pontuações mais altas } \\
\text { nas respostas An, MOR, } \\
\text { Sx, e FQ-, em vítimas de } \\
\text { abuso sexual. }\end{array}$ \\
\hline
\end{tabular}

\section{Câncer e outras doenças}

\begin{tabular}{|c|c|c|c|c|}
\hline $\begin{array}{l}\text { Balottin et al., } \\
\text { 2018/ Itália }\end{array}$ & $\begin{array}{c}\text { Investigar características } \\
\text { de personalidade com o } \\
\text { uso do RSC e a regulação } \\
\text { da emoção e estratégias } \\
\text { de enfrentamento, em } \\
\text { pacientes adolescentes } \\
\text { com enxaqueca, } \\
\text { comparando-os com } \\
\text { adolescentes com } \\
\text { epilepsia idiopática e } \\
\text { adolescentes saudáveis. }\end{array}$ & $\begin{array}{c}51 \text { adolescentes, } \\
\text { sendo } 21 \text { pacientes } \\
\text { com enxaqueca, } \\
20 \text { pacientes } \\
\text { com epilepsia e } \\
11 \text { adolescentes } \\
\text { saudáveis, entre } 11 \\
\text { e } 17 \text { anos de idade } \\
(\mathrm{M}=14 \text { anos). }\end{array}$ & $\begin{array}{c}\text { CDI, CF+C > FC, } \\
\mathrm{D}<0, \text { PER, MOR, } \\
\text { FD, PSV, DQv, } \\
\text { WSum6. }\end{array}$ & $\begin{array}{c}\text { Houve aumento } \\
\text { significativo nas variáveis } \\
\text { CDI, CF+C > FC, PER, } \\
\text { MOR, FD, PSV, DQv, } \\
\text { WSum6 no grupo de } \\
\text { adolescentes com } \\
\text { enxaqueca, comparado } \\
\text { com pacientes com } \\
\text { epilepsia e o grupo } \\
\text { controle. }\end{array}$ \\
\hline
\end{tabular}




\begin{tabular}{|c|c|c|c|c|}
\hline $\begin{array}{c}\text { Autor/ano/ } \\
\text { local }\end{array}$ & Objetivo & População & Variáveis & Resultados \\
\hline \multicolumn{5}{|c|}{ Câncer e outras doenças } \\
\hline $\begin{array}{l}\text { Signorelli, } \\
2015 \text { / } \\
\text { Argentina }\end{array}$ & $\begin{array}{l}\text { Verificar indicadores das } \\
\text { respostas no Rorschach, } \\
\text { Escola Francesa, de } \\
\text { mulheres diagnosticadas } \\
\text { com diferentes tipos } \\
\text { de câncer, utilizando } \\
\text { a fenomenologia } \\
\text { estrutural. }\end{array}$ & $\begin{array}{c}15 \text { mulheres, } \\
\text { entre } 36 \text { e } 66 \\
\text { anos de idade, } \\
\text { diagnosticadas } \\
\text { com diferentes } \\
\text { tipos de câncer: } \\
52 \% \text { tinham } \\
\text { câncer de mama, } \\
27 \% \text { estavam em } \\
\text { tratamento, } 33 \% \\
\text { faziam controle } \\
\text { do tratamento e } \\
27 \% \text { não tinham a } \\
\text { doença. }\end{array}$ & $\begin{array}{c}\text { C', imprecisão na } \\
\text { forma, textura e } \\
\text { Mp. }\end{array}$ & $\begin{array}{c}\text { As participantes } \\
\text { responderam com figuras } \\
\text { pobremente definidas; } \\
\text { utilizaram a cor preta com } \\
\text { conotações depressivas; } \\
\text { fizeram referências ao } \\
\text { corpo relacionado à } \\
\text { doença. }\end{array}$ \\
\hline $\begin{array}{l}\text { Lis, Mazzeschi, } \\
\text { Di Riso, \& } \\
\text { Salcuni, 2011/ } \\
\text { Itália }\end{array}$ & $\begin{array}{l}\text { Abordar questões sobre } \\
\text { o lugar do apego } \\
\text { no funcionamento da } \\
\text { personalidade de um } \\
\text { paciente, com o uso } \\
\text { do RSC; apresentar o } \\
\text { Sistema de Imagem } \\
\text { Projetiva de Apego para } \\
\text { Adultos (AAP) como uma } \\
\text { medida de avaliação } \\
\text { válida e produtiva. }\end{array}$ & $\begin{array}{l}\text { Estudo de caso, } \\
\text { adolescente de } \\
17 \text { anos com } \\
\text { anorexia. }\end{array}$ & $\begin{array}{c}\text { FC:CF+C; } \\
\text { SumC':WSumC; } \\
\text { SumH; GHR:PHR; } \\
\text { XA\%. }\end{array}$ & $\begin{array}{l}\text { Constatou-se controle das } \\
\text { expressões emocionais } \\
\text { (FC:CF + C = 1:0), } \\
\text { e da manifestação } \\
\text { dos sentimentos } \\
\text { (SumC':WSumC = 4:0.5), } \\
\text { baixa autoestima } \\
\text { e autoconfiança, } \\
\text { dificuldade em perceber a } \\
\text { realidade (XA\% = .65). }\end{array}$ \\
\hline $\begin{array}{l}\text { Appel, } 2011 / \\
\text { Israel }\end{array}$ & $\begin{array}{c}\text { Examinar a relação } \\
\text { dialética entre realidade } \\
\text { e fantasia e sua aplicação } \\
\text { à psicossomática, } \\
\text { com o uso do RSC em } \\
\text { adolescentes. }\end{array}$ & $\begin{array}{l}2 \text { adolescentes, de } \\
14 \text { anos de idade, } \\
\text { com sintomas } \\
\text { psicossomáticos. }\end{array}$ & $\begin{array}{c}\text { An + Xy; M; } \\
\text { FC; EA; Blends; } \\
\text { a:p; INCOM; } \\
\text { FABCOM; ALOG; } \\
\text { Sum6; WSum6; } \\
\text { DR; DV; }\end{array}$ & $\begin{array}{c}\text { Pacientes psicossomáticos } \\
\text { podem apresentar } \\
\text { psicopatologias } \\
\text { diferentes. Um paciente } \\
\text { apresentou alexitimia, } \\
\text { respostas no RSC simples, } \\
\text { sem aprofundamento, } \\
\text { Sum6 = 2, a:p = 0:4, } \\
\text { Ma:Mp = 0:3; outro } \\
\text { paciente não apresentou } \\
\text { alexitimia, mas mostrou } \\
\text { índice elevado de } \\
\text { WSum6 = 38, devido à } \\
\text { tendência de combinar } \\
\text { objetos, ideias e } \\
\text { impressões de formas } \\
\text { bizarras (INCOM, } \\
\text { FABCOM, ALOG). }\end{array}$ \\
\hline
\end{tabular}




\begin{tabular}{|c|c|c|c|c|}
\hline $\begin{array}{c}\text { Autor/ano/ } \\
\text { local }\end{array}$ & Objetivo & População & Variáveis & Resultados \\
\hline \multicolumn{5}{|c|}{ Câncer e outras doenças } \\
\hline $\begin{array}{l}\text { Yamamoto } \\
\text { et al., } 2010 \text { / } \\
\text { Japão }\end{array}$ & $\begin{array}{c}\text { Avaliar funções e } \\
\text { processos psicológicos } \\
\text { abrangentes em } \\
\text { pacientes japoneses com } \\
\text { dor crônica, com o uso } \\
\text { do RSC. }\end{array}$ & $\begin{array}{l}48 \text { pacientes com } \\
\text { dor crônica e } \\
\text { média de idade } \\
\text { de } 43 \text { anos, e um } \\
\text { grupo controle, } \\
\text { composto por } \\
200 \text { homens e } 200 \\
\text { mulheres com } \\
\text { média de idade de } \\
35 \text { anos. }\end{array}$ & $\begin{array}{l}\text { Dd, Zd, PSV, PTI, } \\
\text { X-\%, FQ-. S-, } \\
\text { MOR, Wsum6: } \\
\text { DV, DR, INCOM, } \\
\text { FABCOM, ALOG, } \\
\text { CONTAM. }\end{array}$ & $\begin{array}{c}\text { Foram encontrados } \\
\text { aumentos significativos } \\
\text { nos indicadores: } \\
\text { Controle de estresse: Es. } \\
\text { Afeto: Sum Sh, SumC', } \\
\text { SumY, CF, Blends, Color- } \\
\text { Sh Blends. } \\
\text { Processamento de } \\
\text { informação: Dd, Zd, PSV. } \\
\text { Mediação: X-\%, FQx-\%, S-. } \\
\text { Ideação: MOR, WSum6, } \\
\text { DV, DR, ALOG. } \\
\text { Autopercepção: } \\
\text { SumV, MOR. } \\
\text { Relacionamento } \\
\text { interpessoal: PHR, } \\
\text { AG, PER. }\end{array}$ \\
\hline $\begin{array}{c}\text { Porcelli, } 2010 \text { / } \\
\text { Italia }\end{array}$ & $\begin{array}{l}\text { Avaliar os indicadores } \\
\text { do RSC para sofrimento } \\
\text { biopsicológico em } \\
\text { pacientes com doença } \\
\text { inflamatória intestinal } \\
\text { crônica }\end{array}$ & $\begin{array}{c}92 \text { pacientes } \\
\text { com doença } \\
\text { inflamatória } \\
\text { intestinal crônica } \\
\text { com média de } \\
\text { idade de } 36 \text { anos. }\end{array}$ & $\begin{array}{c}\text { D, Adj.D, EA, } \\
\text { m, SumY; DEPI; } \\
\text { Blends, Afr, } \\
\text { An+Xy, PER, Sc, } \\
\text { D. }\end{array}$ & $\begin{array}{l}\text { Os indicadores D / Adj.D } \\
\text { e SumY mostraram-se } \\
\text { confiáveis e estáveis para } \\
\text { experiências estressantes } \\
\text { e a expressão de } \\
\text { componentes somáticos. }\end{array}$ \\
\hline $\begin{array}{c}\text { Flahault, } 2010 \\
\text { / França }\end{array}$ & $\begin{array}{l}\text { Avaliar adolescentes } \\
\text { antes de realizarem } \\
\text { transplante de pulmão, } \\
\text { utilizando-se do RSC. }\end{array}$ & $\begin{array}{c}2 \text { pacientes de } 17 \\
\text { e } 20 \text { anos, com } \\
\text { fibrose cística. }\end{array}$ & $\begin{array}{c}\text { CDI; DEPI; FD; } \\
\text { MOR; FC > CF + } \\
\text { C; COP; C'; } \mathrm{Xu} \% ; \\
\text { P, D. }\end{array}$ & $\begin{array}{l}\text { Um dos adolescentes } \\
\text { revelou elevação no } \\
\text { número de respostas P, } \\
\text { Xu\% e respostas MOR; } \\
\text { a outra adolescente } \\
\text { evidenciou elevação } \\
\text { no número de } \\
\text { respostas D, Xu\%. }\end{array}$ \\
\hline
\end{tabular}

Nota: $(\mathrm{H})=$ Pára-humano inteiro; $(\mathrm{Hd})=$ Detalhe pára-humano; a:p= ativo:passivo; Adj.D= Nota D ajustada; Afr= Quociente Afetivo; $\mathrm{AG}=$ Respostas com movimento agressivo; $\mathrm{ALOG}=$ Respostas com lógica inadequada; $\mathrm{An}=\mathrm{Anatomia} ; \mathrm{Bl}=\mathrm{Sangue}$; $C^{\prime}=$ Resposta cor acromática; $\mathrm{C}=$ Respostas determinante cor; $\mathrm{CDI}=$ Índice de déficit relacional; $\mathrm{CF}=\mathrm{Respostas}$ determinante cor sobre forma; $\mathrm{COP}=$ Movimento cooperativo; CONTAM= Contaminação; $\mathrm{D}=$ Resposta usual; Dd= Resposta inusual em parte da mancha; DEPI= Índice de depressão; DQv= Resposta de qualidade vaga; DR= Resposta desviante; DV= Verbalização desviante; EgoIndex= Índice de egocentrismo; FABCOM= Combinação fabulada; FC= Respostas determinante forma sobre cor; $\mathrm{FD}=$ Respostas de profundidade; $\mathrm{FQ}-=$ Qualidade formal menos; $\mathrm{GHR}=\mathrm{Boa}$ representação humana; H= Humano inteiro; $\mathrm{Hd}=$ Detalhe humano; INCOM= Combinação incongruente; $\mathrm{M}=$ Movimento humano; $\mathrm{m}=\mathrm{Movimento}$ inanimado; $\mathrm{MOR}=$ conteúdo mórbido; $\mathrm{Mp}=$ Movimento passivo; $\mathrm{PER}=$ Respostas personalizadas; $\mathrm{PHR}=\mathrm{Má}$ representação humana; PSV= Respostas com perseveração; PTI= Índice de transtornos de percepção e pensamento; RSC= Rorschach no Sistema Compreensivo; S-= Respostas de distorção do espaço branco; S= Respostas ao espaço branco; Score Z= Atividade organizativa; FM= Respostas de movimento animal; Sum6= Somatório de códigos especiais; SumC'= Somatório de respostas de cores acromáticas; SumC= Somatório de respostas de cor cromática; SumH= Somatório respostas conteúdo humano; SumT= Somatório de respostas de textura; SumV= Somatório de respostas sombreado vista; SumY= Somatório de respostas sombreado difuso; $\mathrm{Sx}=\mathrm{Sexo}$; WSum6= Soma ponderada de códigos especiais; WSumC= Soma ponderada respostas de cor; $\mathrm{X}-\%=$ Forma distorcida; $\mathrm{XA} \%=$ Soma das respostas de qualidade formal adequadas; $\mathrm{Xo} \%=$ Forma convencional; $\mathrm{Xu} \%=\mathrm{Forma}$ incomum; $\mathrm{Xy}=$ Raio $\mathrm{x} ; \mathrm{Zd}=$ Nota $\mathrm{Z}$. 
Verifica-se na Tabela 1 que os estudos da amostra estudada contemplaram de um a 157 indivíduos. A maioria dos estudos sobre vítimas de violência sexual foram compostos por crianças e adolescentes com idades entre 6 e 17 anos. Nota-se que, até o presente momento, não há estudos sobre pacientes infantis com câncer avaliados pelo método de Rorschach, mas somente com pacientes adolescentes e adultos.

Os estudos utilizaram o método de RSC com o objetivo de avaliar a capacidade do instrumento para identificar vítimas de abuso sexual e quais indicadores são responsáveis por essa diferenciação. Também houve avaliações comparativas com outros testes, a fim de verificar as capacidades dos instrumentos para indicar a severidade do abuso. Em relação ao câncer e outras doenças, o método de RSC foi utilizado para avaliar características da personalidade e buscar indicadores de diferentes condições clínicas.

A seguir, a Tabela 2 reúne indicadores do método de Rorschach que se destacaram na avaliação de grupos de vítimas de abuso sexual infantil e grupos de pessoas com câncer e outras doenças do corpo. Elencou-se as variáveis presentes em ambos os grupos (abuso sexual infantil e câncer e outras doenças), pelo menos uma vez em cada grupo, e buscou-se identificar possíveis semelhanças entre eles.

\section{Tabela 2}

Descrição dos indicadores do Rorschach presentes em vítimas de abuso sexual infantil e pacientes com câncer e outras doenças do corpo.

\begin{tabular}{|c|c|c|c|}
\hline Grupo & Autor e ano & Indicadores que se destacaram & $\begin{array}{c}\text { Indicadores presentes em } \\
\text { ambos os grupos }\end{array}$ \\
\hline \multirow[t]{4}{*}{$\begin{array}{l}\text { Abuso sexual } \\
\text { infantil }\end{array}$} & $\begin{array}{l}\text { Scortegagna \& Villemor- } \\
\quad \text { Amaral, } 2013\end{array}$ & $\mathrm{~m}, \mathrm{Bl}, \mathrm{MOR}$ & MOR \\
\hline & $\begin{array}{l}\text { Barnett, Heinze, \& Arble, } \\
2013\end{array}$ & $\mathrm{Bl}, \mathrm{Sx}, \mathrm{An}, \mathrm{MOR}, \mathrm{AG}$ & MOR, AG \\
\hline & $\begin{array}{l}\text { Perfect, Tharinger, Keith, \& } \\
\text { Lyle-Lahroud, } 2011\end{array}$ & $\begin{array}{l}\text { MOR, PER, SumY, SumC', PTI, } \\
\text { GHR, PHR e T }\end{array}$ & $\begin{array}{l}\text { MOR, PER, SumY, SumC', } \\
\text { PHR e T }\end{array}$ \\
\hline & $\begin{array}{l}\text { Scortegagna \& Villemor- } \\
\quad \text { Amaral, } 2009\end{array}$ & $\begin{array}{c}\text { m, Bl, An, MOR, Sx, e de qualidade } \\
\text { formal FQ-. }\end{array}$ & MOR, FQ- \\
\hline \multirow{7}{*}{$\begin{array}{c}\text { Câncer e } \\
\text { outras doenças } \\
\text { do corpo }\end{array}$} & Balottin et al., 2018 & $\begin{array}{c}\text { CDI, CF+C > FC, PER, MOR, FD, } \\
\text { PSV, DQv, WSum6 }\end{array}$ & PER, MOR \\
\hline & Signorelli, 2015 & SumC', Mp, Textura, & SumC', T \\
\hline & $\begin{array}{l}\text { Lis, Mazzeschi, Di Riso, \& } \\
\text { Salcuni, } 2011\end{array}$ & * & * \\
\hline & Appel, 2011 & * & * \\
\hline & Yamamoto et al., 2010 & $\begin{array}{c}\text { Es, Sum Shading, SumC', SumY, } \\
\text { CF, Blends, Color-Shading Blends, } \\
\text { Dd, Zd, PSV, X-\%, FQ-, S-, MOR, } \\
\text { WSum6, DV, DR, ALOG, SumV, } \\
\text { PHR, AG, PER }\end{array}$ & $\begin{array}{l}\text { SumC', SumY, MOR, PHR, } \\
\text { AG, PER, FQ- }\end{array}$ \\
\hline & Porcelli, 2010 & D / Adj.D e SumY & SumY \\
\hline & Flahault, 2010 & Xu\%, P, MOR, D & MOR \\
\hline
\end{tabular}

Nota: *Não apresentou aumentos significativos. Os códigos estão descritos nas notas da Tabela 1. 
Verifica-se na Tabela 2 que a variável MOR apresentou maior frequência $(n=7)$, na medida em que esteve presente em estudos sobre vítimas de abuso sexual $(n=4)$ e sobre pacientes com câncer e outras doenças $(n=3)$. Na sequência, as variáveis PER, SumY e SumC' mostraram-se frequentes $(n=3)$ em estudos sobre pacientes com câncer e outras doenças $(n=2)$ e em estudo sobre vítimas de abuso sexual $(n=1)$. Por fim, as variáveis AG, PHR, FQ- e T foram frequentes $(\mathrm{n}=2)$, com incidência em estudos sobre pacientes com câncer e outras doenças $(n=1)$ e em estudo sobre vítimas de abuso sexual $(\mathrm{n}=1)$. A seguir, a Tabela 3 apresenta as diferenças encontradas nos indicadores do Rorschach nas temáticas pesquisadas.

Tabela 3

Indicadores com potencial diferenciativo de abuso sexual infantil e câncer e/ou outras doenças, encontradas nos estudos.

\begin{tabular}{ccc}
\hline Indicadores & Abuso sexual infantil & Câncer e/ou outras doenças \\
\hline Processamento & $*$ & Zd, DQv, PSV e Dd \\
Mediação & $*$ & P, S-, X-\% e Xu\% \\
Ideação & m e PTI & Mp, WSum6, ALOG, DV, DR \\
Afeto & $*$ & CF+C > FC, SumV \\
Relacionamento interpessoal & GHR & CDI, FD \\
Controle e tolerância & $*$ & D, AdjD, es \\
Autopercepção & An & $*$ \\
Índice traumático & Bl e Sx & $*$ \\
\hline
\end{tabular}

Nota. * Não possuem variáveis. Descrição das variáveis encontra-se na Tabela 1.

Verifica-se na Tabela 3 que, nos estudos selecionados, os indicadores que se destacaram e que podem diferenciar vítimas de abuso sexual infantil foram $\mathrm{Bl}$ $(\mathrm{n}=3)$, Sx $(\mathrm{n}=2), \mathrm{m}(\mathrm{n}=2)$, An ( $=2)$, PTI $(\mathrm{n}=1)$, GHR $(\mathrm{n}=1)$. Os que diferenciaram pacientes com câncer ou outras doenças concentram-se nos módulos de ideação $(n=5)$, mediação $(n=4)$ e processamento $(n=4)$.

\section{Discussão}

Após a identificação das variáveis mais frequentes em ambos os grupos e das variáveis discrepantes do método de Rorschach nas avaliações de vítimas de abuso sexual e de pacientes com câncer e enfermidades físicas (Tabelas 2 e 3), interpretou-se os dados obtidos, que foram reunidos em três conjuntos de variáveis. O primeiro conjunto é composto pelas variáveis mais frequentes nas avaliações de vítimas de abuso sexual e de pacientes com câncer e outras doenças físicas; o segundo é ordenado por variáveis que diferiram nas vítimas de abuso sexual quando comparadas a pacientes com câncer ou outras doenças físicas; e o terceiro compõe-se pelas variáveis que diferiram nos pacientes com câncer e outras doenças físicas, quando comparados às vítimas de abuso sexual.

Observa-se que vítimas de abuso sexual infantil e pacientes com câncer ou outras doenças do corpo podem apresentar semelhanças no funcionamento psíquico reveladas pelo método de RSC. No primeiro conjunto tem-se as variáveis MOR, AG, PER, SumY, SumC', PHR, FQ- e T.

Constata-se, na Tabela 2, que a variável com maior incidência é a MOR, que é considerada como bom suporte empírico na metanálise de Mihura et al. (2013) e que, quando se mostra elevada, sugere autopercepção negativa e distorcida de si, e é sugestiva de sintomas depressivos e de pensamentos pessimistas. Estudos relacionados sobre vítimas de abuso sexual acedem quanto à identificação com um corpo danificado, sofrido ou em ruínas, o que configura uma autoimagem danificada (MOR) decorrente dos maus-tratos sofridos (Perfect et al., 2011; Scortegagna et al., 2016; Scortegagna \& Villemor-Amaral, 2009, 2013).

Em contrapartida, estudos sobre pacientes com câncer e outras doenças não são conclusivos quanto à presença da variável MOR. Enquanto alguns autores 
corroboram estudos prévios e sugerem que o paciente se identifica com um objeto morto, destruído ou em ruínas, em uma autopercepção desqualificante (Balottin et al., 2018), outros estudiosos relacionam a variável MOR apenas à autoimagem negativa, autodepreciativa, que nem sempre está apoiada na realidade (Flahault, 2010; Yamamoto et al., 2010). Essa diferenciação ancora-se na natureza da situação traumática que pode gerar impactos negativos com diferentes níveis de gravidade no psiquismo dos sujeitos. Enquanto alguns sujeitos podem apresentar processos identificatórios mais profundos, em que os conteúdos mórbidos parecem espelhar os resultados dos eventos traumáticos, para outros, os conteúdos mórbidos parecem revelar aspectos de autocrítica.

Como exemplos do efeito traumático do abuso sexual e da identificação das vítimas com corpos danificados, mortos ou em ruínas, observa-se as seguintes respostas com conteúdo MOR encontradas em estudos com crianças vítimas de abuso sexual (Scortegagna et al., 2016, p. 218): "Um homem morto"; "estou vendo igualzinho camisa, é camisa estragada, porque a mulher dele rasga"; "um bicho, que come a cabeça de coelho... o tio matou ele, tá morto"; "cachorro, um bicho matou o cachorro"; "jacaré... mas ele não consegue comer, porque está com a boca costurada”; "osso que quebrou... tá quebrado". As imagens evocadas, como "morto", "estragada", "rasgada", "quebrado" e "boca costurada", testemunham o dano corporal e psíquico de um corpo extinguido. Tais achados coadunam as narrativas "cachorro atropelado", "sapo esmagado", "rato esmagado", "morcego esmagado" e "bichos mortos" verificadas nos estudos de Scortegagna e Villemor-Amaral (2009, 2013), segundo os quais a alta frequência de objetos esmagados revela um corpo bidimensional, sem conteúdo, esvaziado.

Em pacientes com fibrose cística (Flahault, 2010), o conteúdo MOR parece estar mais vinculado ao dano da doença: "Parece um cordeiro... Podemos ver a cabeça claramente e parece estar ferida... por causa do sangue e não está completo" (p. 176). Nesse caso, a ferida na cabeça parece relacionar-se com a condição debilitante do paciente, que não se sente completo por necessitar de máquinas para respirar e por esperar por um transplante, de modo que sua própria ferida foi representada na resposta. Assim, percebe-se que o conteúdo mórbido ancora-se em uma autoimagem depreciativa.

No estudo de Balottin et al. (2018), o aumento do nível de respostas MOR sugeriu que as inseguranças dos adolescentes com enxaqueca podem estar relacionadas a uma percepção negativa e deficiente de si. Os autores consideraram que as respostas MOR dadas por esses adolecentes representavam uma autopercepção deteriorada tanto em nível afetivo quanto ideativo, e identificada com objetos destruídos, mortos ou danificados.

As variáveis PER, PHR, T e AG relacionam-se às percepções dos outros e às características de relacionamento interpessoal. Para vítimas de abuso sexual, esse agrupamento de variáveis pode indicar comportamentos interpessoais menos adaptativos, denotar dificuldades em diferenciar intenções positivas ou negativas e incrementar o risco de reincidência da vitimização (Perfect et al., 2011). Nesse sentido, pacientes com câncer ou outras doenças físicas nem sempre são socialmente comprometidos, mas podem focar em seus aspectos menos positivos, situação em que sentimentos de desamparo e de menor autoeficácia podem predispor sentimentos depressivos, busca por espaço pessoal e atitudes defensivas quando tentam criar novos relacionamentos ou manter os já existentes (Yamamoto et al., 2010).

As variáveis SumC' e SumY conectam-se com o modo como as emoções e os afetos são expressos. Com base em estudos distintos, Schaefer et al. (2018) e Signorelli (2015) corroboram que sintomas depressivos, aflição e ansiedade podem ser efeitos da vitimização sexual e das doenças do corpo. A presença dessas variáveis pode revelar a existência desses sentimentos negativos e a dificuldade em expressá-los enquanto se lida com as demandas do mundo externo (Nascimento, 2010). É possível formular que, quando os sentimentos negativos se tornam muito dolorosos, tanto vítimas de abuso sexual quanto crianças com doenças podem canalizá-los para o corpo, como resultado do sofrimento emocional.

Destaca-se, assim, a importância que o corpo assume em situações de vitimização ou trauma. Se, para as vítimas de abuso sexual, há uma preocupação com o corpo e distorções na autoimagem (Scortegagna \& Villemor-amaral, 2009, 2013; Scortegagna et al., 2016), para pacientes com câncer, o corpo parece perder os limites, de modo que é representado por formas imprecisas: "borboleta incomum porque não é bonita, sua forma não é bem feita e não tem cor"; "coelhos cobrindo os olhos ou com duas coisas saindo como se fosse de outra dimensão"; "abaixo do tronco, algo"; "cara de gato, mas não combina com 
o corpo"; "morto porque ela não tem corpo"; "torso sem continuação" (Signorelli, 2015). Nesses excertos, o protagonismo do corpo como centralizador dos efeitos traumáticos torna possível entender que ambos os casos podem apresentar um corpo lesado em suas representações.

É possível inferir que a autopercepção negativa compromete os relacionamentos interpessoais, devido ao sentimento de desvalorização pessoal aliada à dificuldade em entender as intenções dos outros, o que compromete a formação de vínculos afetivos e, consequentemente, suscita sentimentos de desamparo e temores, expressos no método de Rorschach. Essas reações, podem culminar em sintomas depressivos, aflição e ansiedade, expressos pelo aumento das variáveis SumC' e SumY (Exner Jr., 1999; Nascimento, 2010).

Vítimas de abuso sexual infantil e pacientes com câncer ou outras doenças do corpo podem apresentar semelhanças no funcionamento psíquico. Assim, é importante ressaltar que, ainda que a entrevista forense tenha papel central na avaliação de vítimas de abuso sexual (Schaefer et al., 2018), necessita-se de outros indicadores e critérios objetivos que as caracterizem como tal.

Nesse sentido, o segundo conjunto, composto pelas variáveis que diferiram nas vítimas de abuso sexual (m, GHR, PTI, An, Bl e Sx), incrementa a utilidade do método de RSC como ferramenta na avaliação nesse contexto. Nota-se que vítimas de abuso sexual infantil asseveraram problemas de ideação (PTI) e preocupações relacionadas ao corpo (An), principalmente derivadas de conteúdos traumáticos (Bl) e de conotação sexual (Sx).

Os conteúdos An e Sx, juntamente com Bl, podem revelar fragilidades importantes decorrentes da vitimização sexual e devem ser especialmente considerados nas avaliações (Barnett, Heinze, \& Arble, 2013; Scortegagna \& Villemor-Amaral, 2009). As narrativas "tá saindo sangue da vagina" e "um pênis com uma camisinha toda estourada" são ilustrativas desses conteúdos (Scortegagna \& Villemor-Amaral, 2009).

Além disso, o aumento de PTI pode estar relacionado à severidade do abuso e aos efeitos destrutivos (Perfect et al., 2011). Os autores referem uma tendência à baixa precisão perceptiva e ao pensamento distorcido à medida que aumenta a gravidade do abuso sexual e sobretudo entre os que experimentaram mais subtipos de maus-tratos.
Por haver relação entre a variável Sx e sintomas depressivos, a preocupação e a lembrança da experiência do abuso expressos no Transtorno de Estresse Pós-Traumático (TEPT) podem culminar na presença de sintomas depressivos e representar um prognóstico ruim (Barnett et al., 2013). Nesses casos, as avaliações psicológicas tornam-se elementares para prover medidas tanto preventivas quanto curativas.

Em relação à variável $\mathrm{m}$, quando associada a $\mathrm{Bl}$, Sx e An, indica que o Ego pode estar fragilizado, sofrendo ameaças e ataques ao corpo sobre os quais não se tem controle. O aumento dessas variáveis é considerado forte indicador de abuso sexual (Scortegagna \& Villemor-Amaral, 2013).

Por fim, o terceiro conjunto é composto pelas variáveis que diferiram nos pacientes com câncer ou outras doenças físicas. Para esses casos já existe diagnóstico prévio, assim, a avaliação pelo método de Rorschach pode assumir objetivos distintos em relação às vítimas de abuso sexual infantil. Observa-se mais variáveis com aumento significativo na tríade cognitiva: processamento (Zd, DQv, PSV e Dd), mediação (P, S-, X-\% e Xu\%) e ideação (Mp, WSum6, ALOG, DV e DR).

De modo geral, esses agrupamentos fornecem informações sobre como o indivíduo recebe e traduz as informações ao seu redor, além de como responde a elas. Indicam a presença lapsos no contato com a realidade (WSum6, ALOG, DV, DR), pensamentos mais individualistas (Xu\%) e problemas em interpretar as ações dos outros (X-\%) (Exner, 1999; Nascimento, 2010).

No processamento da informação, Balottin et al. (2018), ao avaliarem adolescentes com enxaqueca, encontraram aumento de respostas de PSV, FD e DQv. Os autores propõem que esses adolescentes podem ser caracterizados por uma espécie de rigidez mental (PSV) que os impede de modificar seus próprios pensamentos e opiniões com flexibilidade adequada. Nesses pacientes, a presença significativa de PSV, juntamente com a elevada frequência das respostas FD, pode refletir uma imagem negativa de si e um temor do julgamento dos outros, o que pode ser compensado com certezas pessoais, um estilo autoritário e aumento de respostas personalizadas (PER). Foi possível notar ainda que esses adolescentes apresentavam uma capacidade de processamento da informação imatura e menos sofisticada, o que denota uma abordagem da realidade, tanto interna quanto externa, bastante vaga e mal definida (DQv).

Para Yamamoto et al. (2010), os valores altamente significativos de MOR e de WSum6, encontrados na 
avaliação de pacientes com dor crônica, sugerem dificuldades no pensamento conceitual ou de clareza ideacional. A presença de MOR em pacientes com dor crônica pressupõe que eles antecipam resultados sombrios para seus esforços no tratamento e que essa percepção negativa provavelmente será reforçada por uma sensação de desamparo. Aliado a isso, os pacientes com dor crônica podem apresentar tendência de raciocínio não convencional (ALOG) decorrente da percepção da dor como um problema catastrófico, o que contribui para intensificar a experiência de dor e incrementar o sofrimento emocional (Sullivan et al., 2001).

Os problemas na autoimagem de pacientes com diferentes tipos de câncer podem estar relacionados a conexões com a morte, sofrimento e alterações físicas (Signorelli, 2015). Para a autora, as pacientes podem sentir que algo estranho e incompreensível está ocorrendo com seus corpos, o que se configura em um traço remanescente da doença; podem apresentar mais dificuldades em enfrentar a realidade, inclusive em aceitar a própria doença; e podem compelir ao incremento da dependência de pessoas e ao afastamento de atividades rotineiras sociais, escolares e profissionais.

\section{Considerações finais}

Embora haja semelhanças na presença de alguns indicadores do RSC entre vítimas de abuso sexual infantil e pacientes com doenças do corpo, percebe-se que a interpretação dessas variáveis se relaciona notadamente à situação vivenciada. Outros indicadores (m, GHR, PTI, An, Bl e Sx) reforçam essas divergências e tanto as semelhanças quanto as diferenças são sinalizadores importantes para a avaliação psicológica e para os profissionais implicados nessas áreas.

A autopercepção negativa, a dificuldade nos relacionamentos interpessoais e os sentimentos depreciativos, acompanham crianças vítimas de abuso ou de doenças do corpo, mas provêm de contextos distintos - forense e clínico ou da psicologia da saúde. A avaliação psicológica, valendo-se do uso do Método de Rorschach e de outros instrumentos de avaliação condizentes com o contexto, pode responder a essas demandas e tornar-se uma grande aliada para os desfechos necessários.

Quanto às doenças do corpo, este estudo revela-se importante por corroborar Signorelli (2015) quando afirma que em casos de avaliação de pessoas saudáveis, as respostas semelhantes do Rorschach encontradas em seu estudo com mulheres com câncer podem ser um sinal de alerta para a detecção precoce da doença e proporcionar benefícios econômicos aos sistemas públicos de saúde. Se a doença for detectada no estágio inicial, o prognóstico do tratamento pode ser mais promissor e pode haver uma taxa de sobrevivência maior. A detecção precoce da doença pode, ainda, levar a um tratamento bem-sucedido e ser benéfica para as pacientes. Se for aplicado a pacientes diagnosticados com câncer, após o tratamento, em casos de recidiva ou metástase, o método de Rorschach pode beneficiar as pacientes por meio do direcionamento da psicoterapia, para que possam reconstruir a autoimagem corporal danificada.

Embora este estudo tenha contribuído com resultados importantes quanto ao compartilhamento de variáveis do Rorschach e quanto às variáveis discrepantes nos grupos distintos, seus resultados precisam ser considerados com cautela. O número reduzido de artigos localizados na realização deste estudo pode ser a limitação mais relevante a ser considerada. Além disso, a falta de estudos precedentes também merece ser relacionada, assim como a falta de estudos com pacientes infantis com câncer avaliados pelo método de Rorschach, que trariam dados importantes para comparação e o incremento dos achados. Portanto, sugere-se o seguimento de pesquisas específicas que avaliem vítimas de abuso sexual infantil e pacientes infantis e adolescentes com câncer, com o uso de outros sistemas de administração do método de Rorschach, como o R-PAS (Meyer, 2017), ou com métodos de manchas de tinta que partilham algumas similaridades, como o Zulliger, na aplicação R-Otimizada.

\section{Referências}

Appel, L. (2011). The Collapse of Potential Space in Adolescents with Psychosomatic Disorders. Rorschachiana, 32(2), 151-182. https://doi.org/10.1027/1192-5604/a000020

Balottin, L., Mannarini, S., Candeloro, D., Mita, A., Chiappedi, M., \& Balottin, U. (2018). Rorschach Evaluation of Personality and Emotional Characteristics in Adolescents with Migraine Versus Epilepsy and Controls. Frontiers in Neurology, 9, 1-10. https://doi.org/10.3389/fneur.2018.00160 
Barnett, D., Heinze, H. J., \& Arble, E. (2013). Risk, Resilience, and the Rorschach: A Longitudinal Study of Children Who Experienced Sexual Abuse. Journal of Personality Assessment, 95(6), 600-609. https://doi.org/10.1080/0022 3891.2013 .823437

Cadan, D., \& Albanese, L. (2018). Um Olhar Clínico para uma Justiça Cega: uma Análise do Discurso de Psicólogos do Sistema de Justiça. Psicologia: Ciência e Profissão, 38(2), 316-331. https://doi.org/10.1590/1982-3703003582017

Exner Jr., J. E. (1999). Manual de classificação do Rorschach para o sistema compreensivo. Casa do Psicólogo.

Flahault, C. (2010). Psychological Assessment of Two Adolescents with Cystic Fibrosis Awaiting Lung Transplants. Rorschachiana, 31(2), 172-191. https://doi.org/10.1027/1192-5604/a000010

Florentino, B. R. B. (2015). As possíveis consequências do abuso sexual praticado contra crianças e adolescentes. Fractal: Revista de Psicologia, 27(2), 139-144. https://doi.org/10.1590/1984-0292/805

Goggin, E. L., Lansky, S. B., \& Hassanein, K. (1976). Psychological Reactions of Children With Malignancies. Journal of the American Academy of Child Psychiatry, 15(2), 314-325. https://doi.org/10.1016/S0002-7138(09)61490-4

Hohendorff, J. Von, Habigzang, L. F., \& Koller, S. H. (2015). Psicoterapia para crianças e adolescentes vítimas de violência sexual no sistema público: Panorama e alternativas de atendimento. Psicologia: Ciência e Profissão, 35(1), 182-198. https://doi.org/10.1590/1982-3703000202014

Krindges, C. A., Macedo, D. M., \& Habigzang, L. F. (2016). Abuso sexual na infância e suas repercussões na satisfação sexual na idade adulta de mulheres vítimas. Contextos Clínicos, 9(1), 60-71. https://doi.org/10.4013/ ctc.2016.91.05

Lahav, Y., \& Elklit, A. (2016). The cycle of healing - dissociation and attachment during treatment of CSA survivors. Child Abuse and Neglect, 60, 67-76. https://doi.org/10.1016/j.chiabu.2016.09.009

Lima,E., S. (2020). Evidências de validade decritério do Zulliger Aplicação R-Otimizada para avaliaro Relacionamento Interpessoal e a Autoimagem em crianças vítimas de violência sexual - Implicações para o Envelhecimento Saudável [Dissertação de mestrado, Universidade de Passo Fundo]. TEDE UPF.

Lis, A., Mazzeschi, C., Di Riso, D., \& Salcuni, S. (2011). Attachment, Assessment, and Psychological Intervention: A Case Study of Anorexia. Journal of Personality Assessment, 93(5), 434-444. https://doi.org/10.1080/00223891. 2011.594125

Meyer, G. J. (2017). What Rorschach performance can add to assessing and understanding personality. International Journal of Personality Psychology, 3(1), 36-49. https://pdfs.semanticscholar.org/cfd5/ 194c43a03869fcbc6a3e242a4a35a419c9ca.pdf

Meyer, G. J., \& Eblin, J. J. (2012). An Overview of the Rorschach Performance Assessment System (R-PAS). Psychological Injury and Law, 5(2), 107-121. https://doi.org/10.1007/s12207-012-9130-y

Mihura, J. L., Meyer, G. J., Dumitrascu, N., \& Bombel, G. (2013). The validity of individual Rorschach variables: Systematic reviews and meta-analyses of the comprehensive system. Psychological Bulletin, 139(3), 548-605. https://doi.org/10.1037/a0029406

Ministério da Saúde. (2018). Análise epidemiológica da violência sexual contra crianças e adolescentes no Brasil, 2011 a 2017. Boletim Epidemiológico da Secretaria de Vigilância Em Saúde, 49(27), 1-17. http://portalarquivos2. saude.gov.br/images/pdf/2018/junho/25/2018-024.pdf

Moher, D., Liberati, A., Tetzlaff, J., Altman, D., \& The PRISMA Group. (2009). Preferred Reporting Items for Systematic Reviews and Meta-Analyses: The PRISMA Statement. PLoS Med, 6(7), 1-6. https://doi.org/10.1371/journal. pmed. 1000097

Nascimento, R. S. G. F. do. (2010). Sistema Compreensivo do Rorschach: Teoria, pesquisa e normas para a população brasileira. Casa do Psicólogo.

Perfect, M. M., Tharinger, D. J., Keith, T. Z., \& Lyle-Lahroud, T. (2011). Relations Between Minnesota Multiphasic Personality Inventory-A Scales and Rorschach Variables With the Scope and Severity of Maltreatment Among Adolescents. Journal of Personality Assessment, 93(6), 582-591. https://doi.org/10.1080/00223891.2011.608754

Porcelli, P. (2010). Rorschach Comprehensive System Predictors of Biopsychological Distress in Patients with Chronic Disease. Rorschachiana, 31(2), 143-171. https://doi.org/10.1027/1192-5604/a000009

Rossato, L., Santeiro, T. V., Barroso, S. M., \& Scorsolini-Comin, F. (2018). Perfil de atendimentos psicológicos em contextos de violência sexual infantojuvenil: Revisão integrativa da literatura. Contextos Clínicos, 11(3), 297-309. https://doi.org/10.4013/ctc.2018.113.02 
Schaefer, L. S., Brunnet, A. E., Lobo, B. O. M., Carvalho, J. C. N., \& Kristensen, C. H. (2018). Indicadores psicológicos e comportamentais na perícia do abuso sexual infantil. Temas Em Psicologia, 26(3), 1467-1482. https://doi.org/ 10.9788/TP2018.3-12Pt

Scortegagna, S. A., Ribeiro, R. K. S. M., \&Villemor-Amaral, A. E. (2016). New Contributions of the Rorschach Method on the Effects of Sexual Abuse in Childhood. Psychology, 7(2), 215-222. https://doi.org/10.4236/psych.2016.72024

Scortegagna, S. A., \& Villemor-Amaral, A. E. (2009). Autopercepção no Rorschach de vítimas de abuso sexual infantil. Psico, 40(3), 328-336. http://revistaseletronicas.pucrs.br/ojs/index.php/revistapsico/article/view/5736

Scortegagna, S.A., \&Villemor-Amaral,A. E. (2013).Traumaticloss and helplessness : qualitative analysis of responses in the Rorschach. Psico-USF, 18(1), 1-12.http://www.scielo.br/scielo.php?script=sci_arttext\&pid=S1413-82712013000100002

Signorelli, S. C. (2015). Cancer and Rorschach from an Existential Perspective. Existential Analysis, $26(1), 70-85$. https://www.thefreelibrary.com/Cancer+and+Rorschach+from+an+existential+perspective-a0407528552

Sullivan, M., Thorn, B., Haythornthwaite, J., Keefe, F., Martin, M., Bradley, A., \& Lefebvre, J. (2001). Theoretical Perspectives on the Relation Between Catastrophizing and Pain. The Clinical Journal of Pain, 17(1), 52-64. https://doi.org/10.1097/00002508-200103000-00008

Yamamoto, K., Kanbara, K., Mutsuura, H., Ban, I., Mizuno, Y., Abe, T., Yoshino, M., Tajika, A. Nakai, Y., \& Fukunaga, M. (2010). Psychological characteristics of Japanese patients with chronic pain assessed by the Rorschach test. BioPsychoSocial Medicine, 4(1), 1-13. https://doi.org/10.1186/1751-0759-4-20

\section{Eduardo dos Santos de Lima}

Psicólogo, mestre em envelhecimento humano pelo Programa de Pós-Graduação em Envelhecimento Humano da Universidade de Passo Fundo (UPF), Passo Fundo - RS. Brasil.

E-mail: eduardo_sevn@hotmail.com

(D) https://orcid.org/0000-0002-8816-6616

\section{Silvana Alba Scortegagna}

Psicóloga, doutora em psicologia pela Universidade São Francisco, Itatiba - SP. Brasil. Docente do Programa de PósGraduação Stricto Sensu em Envelhecimento Humano da Universidade de Passo Fundo (UPF), Passo Fundo - RS. Brasil.

E-mail: silvanalba@upf.br

(D) http://orcid.org/0000-0002-5100-6459

Endereço para envio de correspondência:

Universidade de Passo Fundo, Campus I. BR 285, Km 171, Bairro São José. CEP: 99052-900, Caixa Postal 611.

Passo Fundo - RS. Brasil.

Recebido31/03/2019

Aceito 11/09/2020

Received 03/31/2019

Approved 09/11/2020

Recibido 31/03/2019

Aceptado 11/09/2020 
Lima, E. S., \& Scortegagna, S. A. (2021). Abuso sexual infantil e doenças avaliados pelo Rorschach.

Como citar: Lima, E. S., \& Scortegagna, S. A. (2021). Abuso Sexual Infantil, Câncer e Outras Doenças Avaliados pelo Rorschach: Revisão Sistemática. Psicologia: Ciência e Profissão, 41, 1-15. https://doi.org/10.1590/1982-3703003222038

How to cite: Lima, E. S., \& Scortegagna, S. A. (2021). Child Sexual Abuse, Cancer, and Other Diseases Assessed by the Rorschach: Systematic Review. Psicologia: Ciência e Profissão, 41, 1-15. https://doi.org/10.1590/1982-3703003222038

Cómo citar: Lima, E. S., \& Scortegagna, S. A. (2021). Abuso Sexual Infantil, Cáncer y Otras Enfermedades Evaluadas por el Rorschach: Revisión Sistemática. Psicologia: Ciência e Profissão, 41, 1-15. https://doi.org/10.1590/1982-3703003222038 